\title{
SCHAUDER DECOMPOSITIONS IN BANACH SPACES ${ }^{1}$
}

\author{
BY WILLIAM J. DAVIS
}

Communicated by B. Yood, June 21, 1968

A sequence $\left(M_{i}\right)$ of closed subspaces of a Banach space $E$ is called a Schauder decomposition of $\left[M_{i}\right]$, the smallest subspace containing $\cup M_{i}$, if every element $u$ of $\left[M_{i}\right]$ has a unique, norm convergent expansion $u=\sum u_{i}$, where $u_{i} \in M_{i}$ for $i=1,2, \ldots$. It is well known (see, e.g. [2]) that any sequence $\left(u_{i}\right) \subset E$ with $0 \neq u_{i} \in M_{i}$ for $i=1,2, \ldots$ is basic (i.e., a basis for its closed linear span). The converse of this statement is not true, but we do derive the following theorem, and mention several corollaries.

THEOREM. Let $\left(M_{i}\right)$ be a sequence of closed subspace of the Banach space $E$ such that each sequence $\left(u_{i}\right) \subset E$ with $0 \neq u_{i} \in M_{i}$ is basic. Then there exists an integer $N$ such that $\left(M_{i} \mid i \geqq N\right)$ is a Schauder decomposition of $\left[M_{i} \mid i \geqq N\right]$.

To simplify the proof of the theorem, we use the following characterization of Schauder decompositions due to Grinblyum [3]. A sequence $\left(M_{i}\right)$ of closed subspaces of $E$ is a Schauder decomposition of $\left[M_{i}\right]$ if and only if there exists a constant $K$ such that for all integers $n, m$ and all sequence $\left(u_{i}\right)$ with $u_{i} \in M_{i},\left\|\sum_{i=1}^{n} u_{i}\right\| \leqq K\left\|\sum_{i=1}^{n+m} u_{i}\right\|$. We note that this norm condition may be replaced by $\left\|\sum_{i=1}^{n} a_{i} u_{i}\right\|$ $\leqq K\left\|\sum_{i=1}^{n+m} a_{i} u_{i}\right\|$ where the scalars $\left(a_{i}\right)$ are also arbitrary. Since a sequence $U=\left(u_{i}\right)$ is basic if and only if there exists $K=K(U)$ such that this last inequality holds for all $\left(a_{i}\right), m$ and $n$, we see that each $\left(u_{i}\right)$ with $u_{i} \in M_{i}$ is basic if $\left(M_{i}\right)$ is a Schauder decomposition.

Let $U=\left(u_{i}\right)$ be a sequence with $0 \neq u_{i} \in M_{i}$, and set $U_{n}=\left(u_{i} \mid i \geqq n\right)$. Let $K\left(U_{n}\right)$ be the smallest constant such that $\left\|\sum_{i=n}^{p} a_{i} u_{i}\right\|$ $\leqq K\left\|\sum_{i=n}^{p+a} a_{i} u_{i}\right\|$ holds for all $K \geqq K\left(U_{n}\right)$, all $\left(a_{i}\right)$ and integers $p, q$.

LEMma. Let $\left(M_{i}\right)$ be a sequence of closed subspaces of $E$ such that each $U=\left(u_{i}\right)$ with $0 \neq u_{i} \in M_{i}$ is basic. Then there exists an integer $N$ and $a$ constant $K \geqq 1$ such that every sequence $U$ as above has $K\left(U_{N}\right) \leqq K$.

Proof. If $K$ and $N$ do not exist, then for each integer $n$ and each $M \geqq 1$, there exists a $U$ with $K\left(U_{n}\right)>M$ (noting $K\left(U_{n+1}\right) \leqq K\left(U_{n}\right)$ ). Choose $U^{(1)}$ so that $K\left(U^{(1)}\right)>2$. Then there exist integers $q_{1}>p_{1}$ such that $\left\|\sum_{j=1}^{p_{1}} a_{j} u_{j}^{(1)}\right\|>2\left\|\sum_{j=1}^{q_{1}} a_{j} u_{j}^{(1)}\right\|$ for some sequence $\left(a_{i}\right)$. Similarly, there exist $U^{(2)}$ and $q_{2}>p_{2}$ such that

1 This work was supported by N.S.F. Grant Number GP-6152. 


$$
\left\|\sum_{j=q_{1}+1}^{p_{1}} a_{i} u_{i}^{(2)}\right\|>4\left\|\sum_{j=q_{1}+1}^{q_{2}} a_{i} u_{i}^{(2)}\right\|,
$$

and in general we get $U^{(j)}$ and integers $p_{j}, q_{j}$ such that $q_{j-1}<p_{j}<q_{j}$ and

$$
\left\|\sum_{i=q_{j-1}+1}^{p_{j}} a_{i} u_{i}^{(j)}\right\|>2^{j}\left\|\sum_{i=q_{j-1}+1}^{q_{j}} a_{i} u_{i}^{(j)}\right\| .
$$

With these bounds, the sequence $U$ defined by $u_{i}=u_{i}^{(j)}$ if $q_{j-i}<i \leqq q_{j}$ is not basic, which is a contradiction proving the lemma.

The theorem follows immediately from the lemma and the Grinblyum criterion.

To see that $N$ is in general greater than 1 , let $E$ be separable, $\left(x_{i}\right)$ a basic sequence in $E$ such that codim $\left[x_{i}\right]=\infty$ and $E_{1}$ a closed subspace of $E$ which is a quasicomplement but not a complement of $\left[x_{i}\right]$ in $E$. (For a construction of such an $E_{1}$ see Gurarii and Kadec [4].) If we set $M_{1}=E_{1}, M_{2}=\left[x_{1}\right], M_{3}=\left[x_{2}\right]$, etc., each sequence with just one element in each $M_{i}$ is basic, but $\left(M_{i}\right)$ is not a Schauder decomposition of $E$ since $M_{1}+\left[x_{i}\right] \subsetneq E$. In order to have $N=1$, then, we must keep $\left[M_{i} \mid i<N\right]$ from being a quasicomplement of $\left[M_{i} \mid i \geqq N\right]$ for each $N$. In fact, the addition of this hypothesis is also sufficient, for then we see that $\left[M_{i}\right]=M_{1} \oplus M_{2} \oplus \cdots \oplus M_{N-1} \oplus\left[M_{i} \mid i \geqq N\right]$, and so $\left(M_{i}\right)$ is a Schauder decomposition of $\left[M_{i}\right]$. These corollaries are now immediate. In each, we let $U$ be an arbitrary sequence $\left(u_{i}\right)$ with $0 \neq u_{i} \in M_{i}$, and call $U$ a proper sequence.

Corollary. $A$ sequence $\left(M_{i}\right)$ of closed subspaces of $E$ is a Schauder decomposition if and only if (a) $\left[M_{i}\right]=\left[M_{i} \mid i<n\right] \oplus\left[M_{i} \mid i \leqq n\right]$ and (b) each proper sequence $U$ is basic.

COROLlARY. The previous corollary holds with (a) replaced by ( $\left.\mathrm{a}^{\prime}\right)$ $\left[M_{i}\right]=M_{k} \oplus\left[M_{i} \mid i \neq k\right]$ for each $k$.

Corollary. Let $\left(M_{i}\right)$ be a sequence of finite-dimensional subspaces of $E$. Then $\left(M_{i}\right)$ is a Schauder decomposition if and only if each proper sequence is basic.

It is easy to see that an $N$ dimensional Banach space $F$ has a basis $\left(f_{i}\right)_{i=1}^{N}$ such that

$$
\left\|\sum_{i=1}^{p} a_{i} f_{i}\right\| \leqq N\left\|\sum_{i=1}^{N} a_{i} f_{i}\right\|
$$


always holds (using, for example, the result of Taylor [5]). The author does not know what the best bound that can replace $N$ in general will be, but it must be greater than 1 (see, e.g. [1]). However, using the last corollary, and the $N$-bound above, we obtain:

Proposition. Let $\operatorname{dim} M_{i} \leqq N, M_{i} \in E$ for $i=1,2, \cdots$, and $\left[M_{i}\right]=E$. Set $N_{j}=\operatorname{dim} M_{1}+\operatorname{dim} M_{2}+\cdots+\operatorname{dim} M_{j}$. The following are equivalent

(a) $\left(M_{i}\right)$ is a Schauder decomposition of $E$,

(b) E has a basis $\left(x_{i}\right)$ with $M_{j}=\left[x_{i} \mid N_{j-1}<i \leqq N_{j}\right]$,

(c) each proper sequence is basic.

The proof of the proposition is a routine exercise.

Problem. Does the previous result hold with the weaker assumption $\operatorname{dim} M_{i}<\infty$ ?

\section{REFERENCES}

1. F. Bohnenblust, Subspaces of $l_{p, n}$ spaces, Amer. J. Math. 63 (1941), 64-72.

2. D. Dean, Schauder decompositions in (m), Proc. Amer. Math. Soc. 18 (1967), 619-623.

3. M. M. Grinbylum, On the representation of a space of type $B$ in the form of $a$ direct sum of subspaces, Dokl. Akad. Nauk SSSR (N.S.) 70 (1950), 749-752. (Russian)

4. V. I. GurariY and M. I. Kadec, Minimal systems and quasicomplements in Banach space, Dokl. Akad. Nauk SSSR 145 (1962), 256-258=Soviet Math. Dokl. 3 (1962), 966-968.

5. A. E. Taylor, $A$ geometric theorem and its application to biorthogonal systems, Bull. Amer. Math. Soc. 53 (1947), 614-616.

Ohio State University 